\title{
Protostellar chemistry dominated by external irradiation
}

\author{
Johan E. Lindberg ${ }^{1}$, Steven B. Charnley ${ }^{1}$, Jes K. Jørgensen ${ }^{2}$, \\ Yoshimasa Watanabe ${ }^{3}$, Suzanne E. Bisschop ${ }^{2}$, Nami Sakai ${ }^{3,4}$, and \\ Satoshi Yamamoto ${ }^{3}$ \\ ${ }^{1}$ NASA Goddard Space Flight Center, \\ Astrochemistry Laboratory, Mail Code 691, \\ 8800 Greenbelt Road, Greenbelt, MD 20771, USA \\ email: johan.lindberg@nasa.gov \\ ${ }^{2}$ Centre for Star and Planet Formation, \\ Niels Bohr Institute and Natural History Museum of Denmark, \\ University of Copenhagen, Øster Voldgade 5-7, DK-1350 Copenhagen K, Denmark \\ ${ }^{3}$ Department of Physics, The University of Tokyo, \\ 7-3-1 Hongo, Bunkyo-ku, Tokyo, 113-0033, Japan \\ ${ }^{4}$ RIKEN, 2-1 Hirosawa, Wako, Saitama 351-0198, Japan
}

\begin{abstract}
Submillimetre observations of externally irradiated low-mass protostellar envelopes show that the gas temperature in the envelopes is dominated by the external irradiation. Detailed studies of the protostar IRS7B in Corona Australis also show that the chemistry is strongly affected by the irradiation, depleting the abundances of complex organic molecules.
\end{abstract}

Keywords. stars: formation, ISM: molecules, astrochemistry, radiative transfer

\section{Introduction}

The low-mass protostars in the $\mathrm{R}$ CrA cloud in the Corona Australis star-forming region $(d=130 \mathrm{pc})$ are externally irradiated by the intermediate-mass protostar $\mathrm{R} \mathrm{CrA}$, which heats the envelopes to $T \gtrsim 30 \mathrm{~K}$ (Lindberg \& Jørgensen 2012).

We conducted spectral line surveys with the ASTE telescope and the APEX telescopet of the Class 0/I protostar IRS7B, irradiated by $\mathrm{R}$ CrA. More details on these two surveys are given by Watanabe et al. (2012) and Lindberg et al. (2015), respectively.

We also used APEX to observe three $\mathrm{H}_{2} \mathrm{CO}$ lines around $218 \mathrm{GHz}$ towards 56 protostellar envelopes in the $\mathrm{CrA}$ and Ophiuchus star-forming regions. The observations were used to measure the rotational temperature of $\mathrm{H}_{2} \mathrm{CO}$, which is a good tracer of the kinetic temperature (Mangum \& Wootten 1993).

\section{Line survey}

In the APEX and ASTE surveys of IRS7B, we detected spectral lines from 22 different molecular species, of which the most complex were $\mathrm{CH}_{3} \mathrm{CHO}, \mathrm{CH}_{3} \mathrm{CCH}, \mathrm{CH}_{3} \mathrm{CN}$, and $\mathrm{CH}_{3} \mathrm{OH}$. The absolute abundance of $\mathrm{CH}_{3} \mathrm{OH}$ in IRS7B is found to be only a few $\times 10^{-9}$, two orders of magnitude lower than the $\mathrm{CH}_{3} \mathrm{OH}$ abundance in the hot corino source IRAS 16293-2422 (Cazaux et al. 2003). Furthermore, the abundance of several other organic molecules (such as $\mathrm{HCOOH}, \mathrm{CH}_{3} \mathrm{CN}, \mathrm{CH}_{3} \mathrm{OCH}_{3}$, and $\mathrm{CH}_{3} \mathrm{OCHO}$ ) relative to $\mathrm{CH}_{3} \mathrm{OH}$ is at least an order of magnitude lower in IRS7B than in IRAS 16293-2422. We propose that a long-term elevated temperature in the protostellar envelopes caused by external irradiation from $\mathrm{R} \mathrm{CrA}$ has led to

$\dagger$ This work is based on observations with the Atacama Pathfinder EXperiment (APEX) telescope. APEX is a collaboration between the Max Planck Institute for Radio Astronomy, the European Southern Observatory, and the Onsala Space Observatory. 


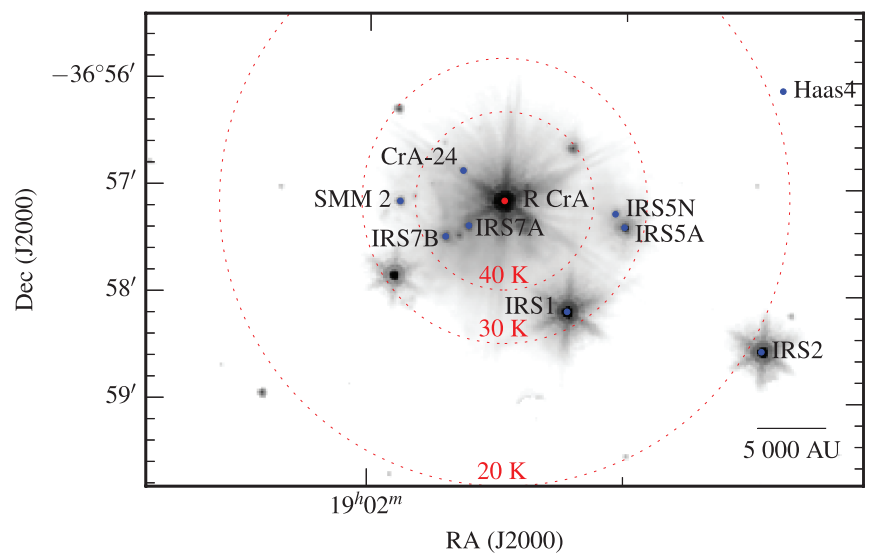

Figure 1. $\mathrm{H}_{2} \mathrm{CO}$ temperatures measured in the protostellar envelopes near the luminous Herbig Be star R CrA.

large-scale evaporation of $\mathrm{CO}$ from the dust grains, inhibiting formation of complex organics. Gas-phase formation of certain complex organic species will also be inhibited due to the low $\mathrm{CH}_{3} \mathrm{OH}$ abundance (Charnley 1997).

\section{3. $\mathrm{H}_{2} \mathrm{CO}$ and $c-\mathrm{C}_{3} \mathrm{H}_{2}$ surveys}

In our survey of $\mathrm{H}_{2} \mathrm{CO}$ and $c-\mathrm{C}_{3} \mathrm{H}_{2}$ in Corona Australis, we find $\mathrm{H}_{2} \mathrm{CO}$ rotational temperatures ranging between $19 \mathrm{~K}$ and $45 \mathrm{~K}$; even higher temperatures are found in two outflow components Lindberg et al. (2015). The difference in temperature is well-correlated with the distance to the luminous $\mathrm{R}$ CrA, and matches with a simple 1-D Transphere (Dullemond et al. 2002) radiative transfer model of the heating from $\mathrm{R} \mathrm{CrA}$. This star heats the gas in the star-forming region on scales of $\sim 30000 \mathrm{AU}$. The $c-\mathrm{C}_{3} \mathrm{H}_{2}$ temperature is, however, found to be $9-17 \mathrm{~K}$ in all sources.

The same trend is seen in the Ophiuchus star-forming region (Lindberg et al., in prep.). We identify the Herbig Be star S 1 and the B2 star HD 147889 as the primary heating sources of the embedded protostars in the $\rho$ Oph A and $\rho$ Oph B clouds, where we find $\mathrm{H}_{2} \mathrm{CO}$ temperatures up to $40 \mathrm{~K}$. The hot corino source IRAS 16293-2422, however, shows the highest $\mathrm{H}_{2} \mathrm{CO}$ temperature in the sample, $73 \mathrm{~K}$, but this emission is thought to originate in the hot inner envelope, with internal irradiation causing the high temperature.

\section{References}

Cazaux, S., Tielens, A. G. G. M., Ceccarelli, C., et al. 2003, ApJL, 593, L51

Charnley, S. B. 1997, in IAU Colloq. 161: Astronomical and Biochemical Origins and the Search for Life in the Universe, ed. C. Batalli Cosmovici, S. Bowyer, \& D. Werthimer, 89

Dullemond, C. P., van Zadelhoff, G. J., \& Natta, A. 2002, A\&GA, 389, 464

Lindberg, J. E. \& Jørgensen, J. K. 2012, A\& A, 548, A24

Lindberg, J. E., Jørgensen, J. K., Watanabe, Y., et al. 2015, A\&A, in press (arXiv:1509.02514)

Mangum, J. G. \& Wootten, A. 1993, ApJS, 89, 123

Watanabe, Y., Sakai, N., Lindberg, J. E., et al. 2012, ApJ, 745, 126 